\title{
MIMO AND COOPERATIVE MIMO COMPARISON IN ENERGY CONSTRAINED WIRELESS SENSOR NETWORKS
}

\author{
Meethu Abraham ${ }^{1}$, Sudhansu Sekhar Singh ${ }^{2}$ \\ ${ }^{I}$ M.Tech Student, School of Electronics Engineering, KIIT University, Bhubaneswar, India \\ ${ }^{2}$ Professor, School of Electronics Engineering, KIIT University, Bhubaneswar, India
}

\begin{abstract}
In Wireless Sensor Network commonly referred as WSN, the hubs or nodes are operated by batteries so that the energy utilization should be diminished, while fulfilling the given throughput and given requirement. The paper studies about the performance and energy consumption of cooperative MIMO and MIMO (multi input multi output) based communication. The average energy usage comprises circuit energy and transmission energy consumption. The comparison between the multi-input- multi-output (MIMO) and cooperative MIMO techniques help us to choose the best scheme for energy constrained wireless sensor network application. The simulation result shows that energy efficiency of MIMO (multi-input-multi-output) and SISO (single-input-single-output) is better for longer distances and thud increase the system life time.
\end{abstract}

Keywords: Cooperative MIMO, MISO, SISO, SIMO, wireless sensor network, energy efficiency, BER performance $* * *$

\section{INTRODUCTION}

In the recent advance in science and technology, the communication system has been marching towards quick development. Wireless sensor network is one of the important blocks for communication. The major limiting factors in wireless sensor network are power consumption requirements, life time of network, data integrity and data confidentiality. A sensor network is a remote system comprising of spatially appropriated self governing sensors to monitor physical or ecological conditions for examples temperature, sound, and so forth and to pass their information through the system to a principle area. Each sensor nodes is accumulation of sensor hardware , microcontrollers, few limit of RAM and program memory, a remote handset and the power supply i.e. battery. The sensor hubs used are small in size and cheap. WSN can be utilized as a part of numerous courses in modern industrial facility computation. Some of the application is monitoring of equipment, military surveillance and machinery health .WSN can be used for leaking or radioactive monitoring in chemical plant. The two main core challenges in WSN are energy efficiency and scalability[1]. In many cases, the replacement of battery is not possible so that energy utilization should be decreased and for the data transfer energy saving transmission scheme should be used in the sensor networks.

With using highest diversity gain the transmission power can be lessened thus lower the transmission rate and increase the reliability. The idea of cooperative MIMO presented in WSN using cooperative nature of sensor node to achieve higher reliability link of communication and lessen transmission puissance. In traditional MIMO, the multiple antenna are appended to one node or other hub but in cooperative MIMO, multiple nodes cooperate to receive or transmit the signal. The numerous hubs or nodes were physically gathered together to receive or transmit .
In [2] it presented that with equal transmit power and similar BER specification MIMO scheme exploits at data rate and thus requires less transmission energy when compared to SISO system. Due to the restricted physical size of node, the direct application of multiple antenna to the system is impossible. The cooperative MIMO can be developed by permitting single antenna node to coordinate on transmission or gathering so that energy efficient scheme can be deployed. The techniques of energy efficiency dissipation mainly concerned on reducing the transmission energy and it used in larger domain applications. On the other hand, in small range applications the consumption of circuit energy is identical which governs the transmission energy. The total energy dissipated in the circuit includes the energy dissipated in circuit blocks with signal way i.e. analog to digital converter (ADC), filter, mixer, low noise amplifier (LNA), frequency synthesizer, intermediate frequency amplifier (IFA) and power amplifier. In MIMO systems though the complexity of the circuit will little higher than that of SISO system but it outperforms it in terms of energy efficiency.

Under same BER ( bit error rate) and throughput, first we compare the energy usage of simple MIMO system with reference SISO systems and then the cooperative MIMO. The energy consumption is compared with the transmission distances.

The remaining part of the paper is organized in the following order. The analytical model for the energy consumption of MIMO system is discuss in the section 2 of the paper while the energy consumption model for cooperative MIMO scheme is explained in section 3. All the attributes consider for the simulation and the obtained results are presented in section 4 . The conclusive remarks and the scope for future work is projected in section 5 . 


\section{ENERGY EFFICIENCY OF MIMO SYSTEMS}

Here, the communication link which connecting between the wireless nodes or hubs may be MIMO (multi input-multioutput), MISO (multiple-input-single-output), SIMO (single-input-multiple-output), or SISO (single-inputmultiple-output). The average energy consumption is calculated considering each signal processing unit at the receiver and the transmitter. In this model, baseband processing signal sections or blocks (i.e. pulse-shaping, source coding and digital modulation) are excluded and error-correction-code (ECC) blocks are also omitted. The figure 1 and 2 represents path of transmitter and receiver side thus $\mathrm{N}_{\mathrm{t}}$ and $\mathrm{N}_{\mathrm{r}}$ denotes the transmitter and receiver antennas respectively[3]. In SISO case, $\mathrm{N}_{\mathrm{tx}}=\mathrm{N}_{\mathrm{rx}}=1$.

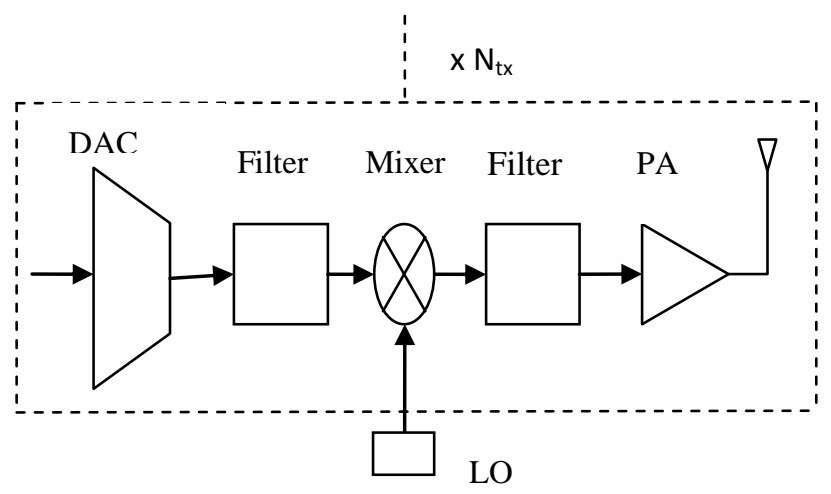

Fig 1. Transmitter Blocks

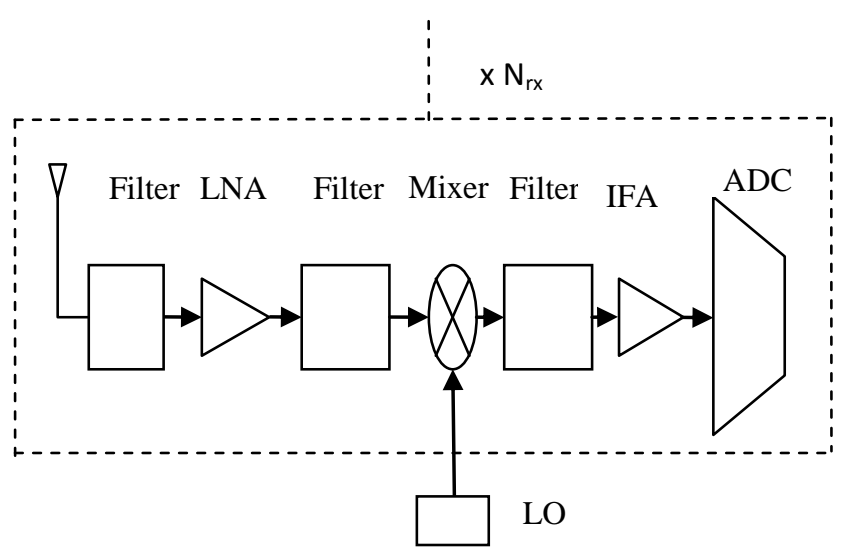

Fig 2. Receiver Blocks

The average power ultization or consumption for signal path is comprises of two primary parts and they are power consumption for the $\mathrm{P}_{\text {AMP }}$ i.e. power amplifier and power consumption for $\mathrm{P}_{\mathrm{C}}$ i.e. circuit blocks. The power amplifier $\mathrm{P}_{\text {AMP }}$ is subject to transmit power $\mathrm{P}_{\text {output }}$ which is described below:

$$
P_{\text {output }}=E_{\text {bit }} R_{\text {bit }} \times \underset{G_{t x} G_{r x} \lambda^{2}}{\left(4 \pi d^{2}\right) M_{1 k}} N_{\text {fk }}
$$

Here $R_{b i t}$ is the bit rate, $E_{b i t}$ is energy per bit for given BER (bit error rate) requirement at receiver side, transmission distance is $d, G_{t x}$ and $G_{r x}$ is transmitter (TX) and receiver (RX) antenna gain respectively, the carrier - wavelength is $\lambda, \mathrm{M}_{\mathrm{lk}}$ is link margin and other added background interference or noise, and then $\mathrm{N}_{\mathrm{fk}}$ is receiver (RX) noise figure defined as $\mathrm{N}_{\mathrm{fk}}=\left(\mathrm{N}_{\mathrm{rk}} / \mathrm{N}_{0}\right)$ and thus $\mathrm{N}_{0}=-172$ $\mathrm{dBm} / \mathrm{Hz}$ thermal noise power spectral density at room temperature and then $\mathrm{N}_{\mathrm{rk}}$ is the power spectral density of the average effective noise seen at receiver input.

The power amplifier average power consumption is

$$
\mathrm{P}_{\mathrm{AMP}}=\left(1+\alpha_{1}\right) \mathrm{P}_{\text {output }}
$$

Here, $\alpha_{1}=\left(\xi_{1} / \eta_{1}\right)-1$, where $\xi_{1}$ is PAR (peak to average ratio) and $\eta_{1}$ is drain efficiency of radio frequency power amplifier.

The power consumption for circuit block $\mathrm{P}_{\mathrm{C}}$ is calculated by

$$
\begin{gathered}
P_{C}=N_{t x}\left(P_{\text {mixer }}+P_{\text {DAC }}+P_{\text {filtx }}\right)+2 P_{\text {sync }}+\underset{\text { rx }}{P_{\text {LNA }}}\left(P_{\text {mixer }}+\right. \\
\left.P_{\text {ADC }}+P_{\text {IFA }}+P_{\text {filrx }}\right)
\end{gathered}
$$

where $\mathrm{P}_{\text {DAC }}, \mathrm{P}_{\text {LNA }}, \mathrm{P}_{\text {ADC }}, \mathrm{P}_{\text {mixer }}, \mathrm{P}_{\text {filtx }}, \mathrm{P}_{\text {IFA }}, \mathrm{P}_{\text {filrx }}, \mathrm{P}_{\text {sync }}$ are power consumption rate for digital to analog converter (DAC) , low noise - amplifier (LNA), analog to digital converter (ADC), the mixer, transmitter side active filters, the intermediate frequency- amplifier (IFA), active filters at receiver-side and frequency-synthesizer, respectively.

The average energy consumption per bit of system can be written by

$$
\mathrm{E}_{\mathrm{btotal}}=\left(\mathrm{P}_{\mathrm{AMP}}+\mathrm{P}_{\mathrm{C}}\right) / \mathrm{R}_{\mathrm{bit}}
$$

The Alamouti scheme are used in this paper to exploit diversity from MIMO schemes. To employ Alamouti code in the MIMO scheme, a pair of antenna are used with two separate symbols s1and s2 which are transmitted all the while amid the first image period from antenna 1 and 2, took after by signs $-\mathrm{s} 2 *$ and $\mathrm{s} 1 *$ from antennas 1 and 2, individually, amid next symbol period. Rayleigh fading channels MIMO scheme based can accomplish lower normal likelihood of mistake than SISO scheme under same transmit energy spending plan because of the diversity gain. At the same bit error rate and throughput prerequisite, multiinput-multi-output(MIMO) schemes require less transmission vitality than single-input-single-output (SISO) schemes.

As per the Chernoff bound (at high SNR- regime)

$$
\mathrm{P}_{\text {bit }} \leq\left(\frac{\text { Ebit }}{\text { Nt No }}\right)^{-\mathrm{Ntx}}
$$

the the upper bound for energy per bit is

$$
\mathrm{E}_{\mathrm{bit}}=\frac{\mathrm{N}_{\mathrm{tx}} \mathrm{N}_{0}}{\mathrm{P}_{\mathrm{bit}}^{1 / \mathrm{Ntx}}}
$$

By using the above bound as equality, we have estimated average energy consumption of MISO scheme and the SISO scheme as per equations (1) and (4). Thus, we can get 


$$
\mathrm{E}_{\mathrm{btotal}}=\left(1+\alpha_{1}\right) \frac{\mathrm{N}_{\mathrm{tx}} \mathrm{N}_{0} \mathrm{x}}{\mathrm{P}_{\mathrm{bit}}^{1 / \mathrm{Ntx}}} \frac{\left(4 \pi \mathrm{d}^{2}\right) \mathrm{M}_{\mathrm{lk}} \mathrm{N}_{\mathrm{fk}}}{\mathrm{G}_{\mathrm{tx}} \mathrm{G}_{\mathrm{rx}} \lambda^{2}} \frac{\mathrm{P}_{\mathrm{C}}}{\mathrm{R}_{\mathrm{bit}}}
$$

\section{MIMO WITH MULTINODE COOPERATION}

The primary concern of the sensor networks is to expand the network lifetime .Since sensor systems are chiefly intended to chip on some -joint task where every hub reasonableness is not underscored, outline plan is to reduce the aggregate energy utilization in system as opposed to reducing energy utilization of individual hubs or nodes. Here, we suggest a methodology for reducing the average energy utilization or consumption of multiple hubs from the system viewpoint.

The information which colllected by numerous neighborhood sensors is transmitted to remote processor in sensor network system. The data collected will be first transmit it to the relay node if remote controller is far away, after that multi - hop based routing is used to send information to its last destination. This situation is outlined in figure 3.

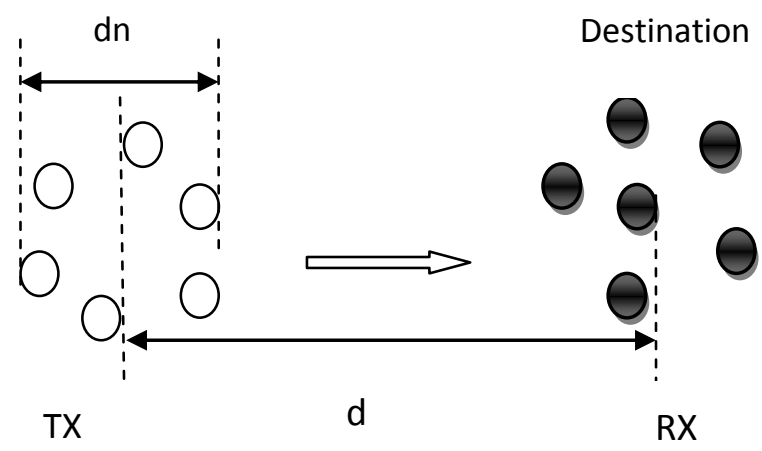

Fig 3. Cooperative MIMO scheme for WSN

The additional amount of energy used for the local cooperative information exchange is based on number of antennas as well as the inter node distance between the cooperative hubs or nodes at transmitting and receiving sides. The inter-node distance is required to fluctuate from 1 to $7 \mathrm{~m}$ based on the geological design of system. We can consider that there is $\mathrm{N}_{\mathrm{bt}}$ bits to broadcast from the hub source to hub destination . Here, we have $\mathrm{N}_{\mathrm{t}}$ number of hubs and $\mathrm{N}_{\mathrm{r}}$ number of hubs to cooperate at transmitter and receiver sides .

At transmitter side, source node broadcasts its $\mathrm{N}_{\mathrm{bt}}$ bits to $\left(\mathrm{N}_{\mathrm{tx}}-1\right)$ agreeable nodes. The SISO scheme is the most energy effective for the short range nearby separation $d_{m}$ .Here expect, there are simply single hop single-inputsingle-output (SISO) scheme transmissions between two agreeable hubs and there an un-coded 16-QAM (quadrature amplitude modulation) is utilized over the channel with $\mathrm{K}$ law path loss considering it only for AWGN environment. The 16 QAM modulation scheme permits the reduction of circuit utilization. According to SISO non co-operative scheme, one can estimate the energy spent per bit for the cooperative transmission $\mathrm{E}_{\mathrm{bcoopTX}}\left(\right.$ for that $\mathrm{d}=\mathrm{d}_{\mathrm{n}}$ and $\left(\mathrm{N}_{\mathrm{tx}}-1\right)$ receiving nodes).
In transmission side, the additional co-operative energy utilization or consumption $\mathrm{E}_{\mathrm{TXbtcoop}}$ is based on energy consumption per bit $\mathrm{E}_{\mathrm{TXbcoop}}$ and estimated as

$$
\mathrm{E}_{\mathrm{TXbtcoop}}=\mathrm{N}_{\mathrm{bt}} \mathrm{E}_{\mathrm{TX} \text { bcoop }}
$$

After getting $\mathrm{N}_{\mathrm{bt}}$ bits from hub $\mathrm{S}$, then cooperative-nodes $\mathrm{N}_{\mathrm{tx}}$ modulates and accordingly they will align their bits to QPSK- STBC images and afterward broadcast all while to the destination hub over the MIMO scheme Rayleigh fading channel.

At the receiver side, then cooperative nodes $\mathrm{N}_{\mathrm{rx}}-1$ firstly gets the MIMO generated symbols and then do quantization for the STBC symbols to $\mathrm{N}_{\mathrm{ss}}$ bit after that it transmit again the quantized bits for the destination hub utilizing un-coded 16 QAM modulation for SISO. At receiver side extra cooperative energy utilization $\mathrm{E}_{\text {btcoopRx }}$ is based on $\mathrm{N}_{\mathrm{rx}}, \mathrm{N}_{\mathrm{ss}}$ and $E_{\mathrm{RXbcoop}}$ the energy consumption of SISO scheme that can be computed by utilizing the SISO 16 QAM transmission scheme for $d_{n}$ distance. $E_{R X b t c o o p}$ is estimated as

$$
\mathrm{E}_{\mathrm{RXbtcoop}}=\mathrm{N}_{\mathrm{ss}}\left(\mathrm{N}_{\mathrm{rx}}-1\right) \mathrm{N}_{\mathrm{bt}} \mathrm{E}_{\mathrm{RXbcoop}}
$$

The average energy consumption of cooperative MIMO is

$$
\mathrm{E}_{\mathrm{TOTAL}}=\mathrm{E}_{\mathrm{btotal}}+\mathrm{E}_{\mathrm{TXbtcoop}}+\mathrm{E}_{\mathrm{RXbtcoop}}
$$

\section{SIMULATION RESULTS}

The result is based on the MATLAB simulation. The simulation were done using the parameters present in the table 1. Findings predicated on simulation utilizing mathematical model provide subsidiary insights into certain performance aspects and identifying promising solutions for the energy-efficient WSNs.

Table 1. System parameters

\begin{tabular}{|l|l|}
\hline $\mathbf{f}_{\text {cr }}=\mathbf{2 . 5} \mathbf{~ G H z}$ & $\boldsymbol{\eta}($ eff $)=\mathbf{0 . 3 5}$ \\
\hline $\mathrm{G}_{\mathrm{tx}} \mathrm{G}_{\mathrm{rx}}=\mathbf{5} \mathbf{d B i}$ & $\mathbf{N}_{\mathbf{0}}=-172 \mathrm{dBm} / \mathrm{Hz}$ \\
\hline $\mathrm{BW}=10 \mathrm{KHz}$ & $\mathrm{Ts}=1 / \mathrm{BW}$ \\
\hline $\mathrm{P}_{\text {mixer }}=30.4 \mathrm{~mW}$ & $\mathrm{P}_{\mathrm{SYNC}}=50.3 \mathrm{~mW}$ \\
\hline $\mathrm{P}_{\mathrm{bt}}=10^{-3}$ & $\mathrm{~B}=1$ \\
\hline $\mathrm{P}_{\text {filtx }}=\mathrm{P}_{\text {filtx }}=2.5 \mathrm{~mW}$ & $\mathrm{P}_{\mathrm{LNA}}=20 \mathrm{~mW}$ \\
\hline $\mathrm{N}_{\mathrm{fk}}=10 \mathrm{~dB}$ & $\mathrm{M}_{\mathrm{Lk}}=40 \mathrm{~dB}$ \\
\hline
\end{tabular}

From figure 4 shows that M-ary Quadrature Amplitude Modulation (MQAM) modulation schemes is preferred for better BER performance when Rayleigh fading is present in wireless sensor networks. 


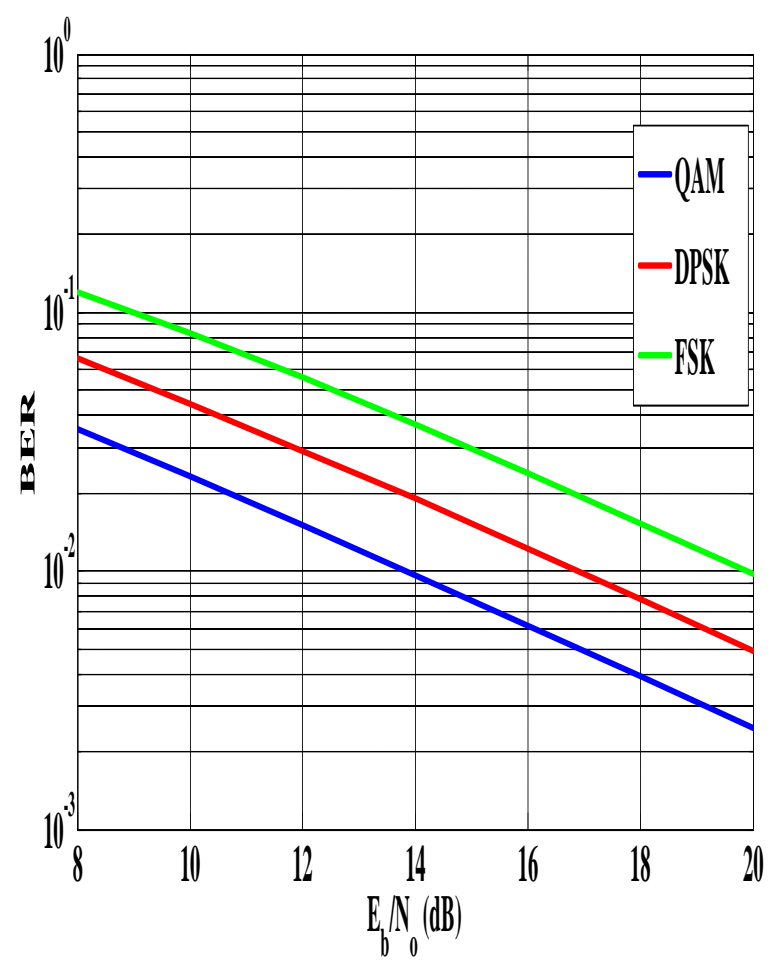

Fig 4. BER performance comparison of different modulation techniques in Rayleigh fading channel

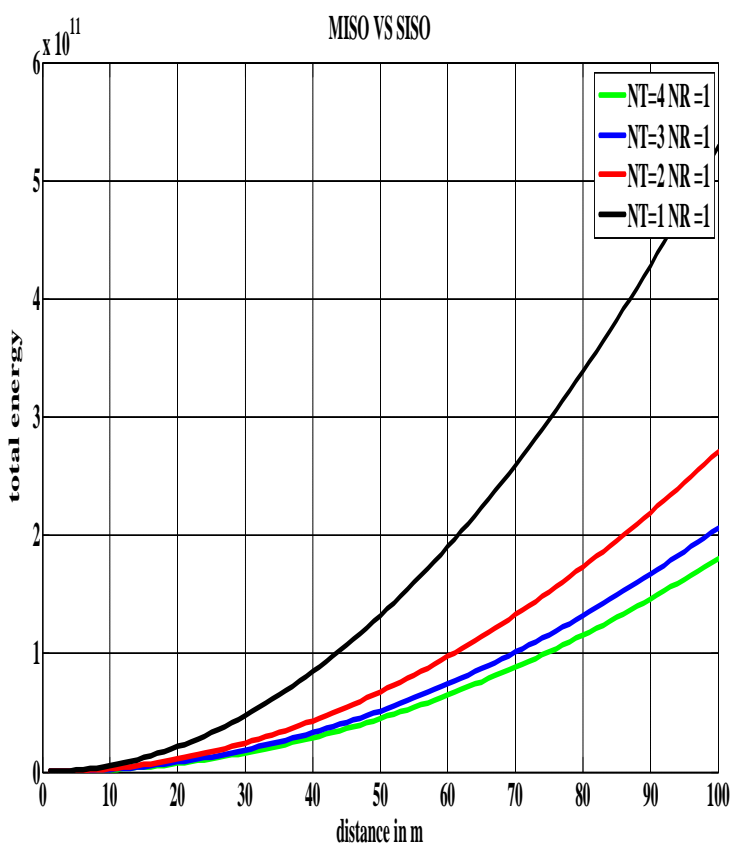

Fig 5. Energy consumption of MISO and SISO system

The figure 5 compares average energy consumption per bit along the transmission distances between the MISO scheme and the SISO scheme in WSN . According to the figure 5, it can analyze MISO scheme requires the less transmission energy for long range application, then average energy consumption will becomes lesser when compared with SISO scheme. When the distances between transmission increases then respectively the energy consumption also increases.

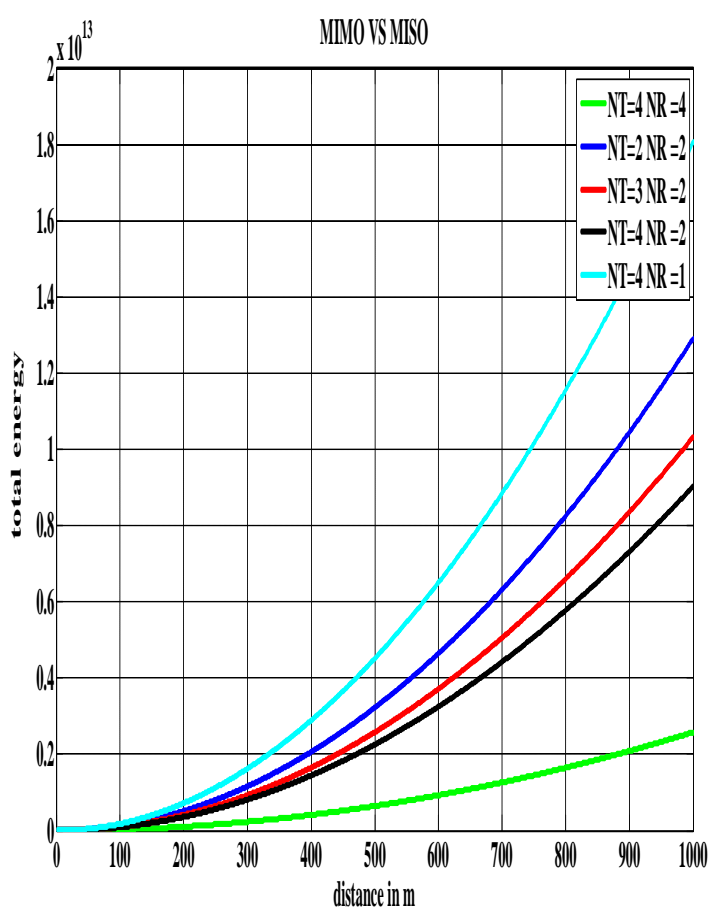

Fig 6. Energy consumption for MISO and MIMO

The figure 6 compares average energy consumption along the transmission distances that between the MIMO scheme and the MISO scheme in WSN .According to the figure 6, it can analyze MIMO scheme requires less transmission energy for long range application, then average energy consumption will becomes lesser when compared with MISO scheme. From the figure we can also determines that incrementing the transmission nodes is a good option than incrementing the receiving nodes.

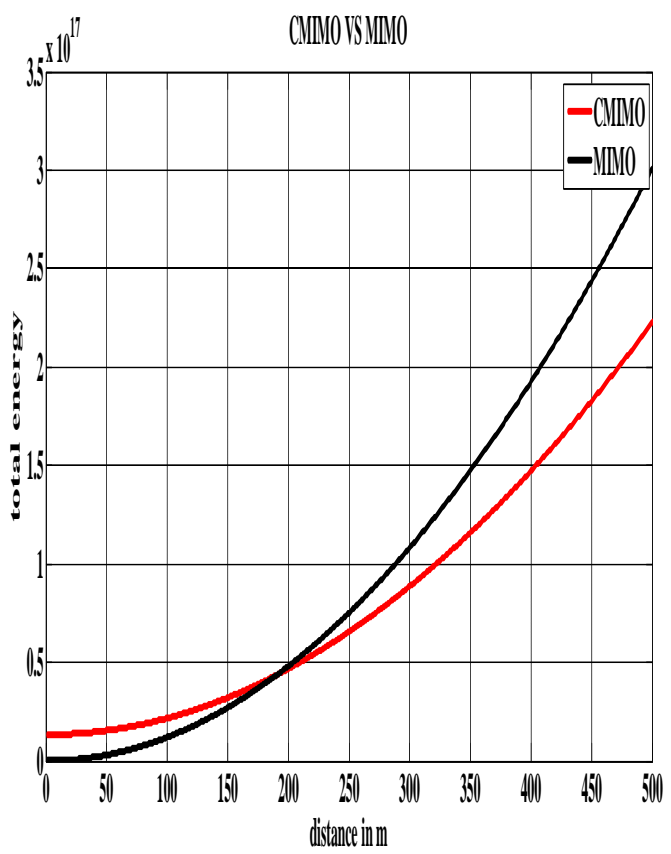

Fig 7. Energy consumption for MIMO and Cooperative MIMO 
The figure 7 compares average energy consumption with the transmission distances between MIMO scheme and cooperative MIMO scheme in WSN. According to the figure 7 , it can analyze cooperative MIMO scheme requires less transmission energy from distance greater than $200 \mathrm{~m}$, then average energy consumption will becomes smaller when compared with MIMO. When the transmission distance is less than $200 \mathrm{~m}$ then the MIMO energy consumption is low.

\section{CONCLUSION}

The paper analyzed the average energy consumption performances of different diversity schemes antennas. The result shows that, Cooperative MIMO performs better than MIMO scheme. And the MISO scheme and MIMO scheme are more energy saving than SISO scheme . For determining the best option of $\mathrm{N}_{\mathrm{tx}}$ and $\mathrm{N}_{\mathrm{rx}}$ for the given transmission distance, the MIMO schemes selection can be performed. The introduction of cooperative MIMO scheme for $2 \times 2$ antennas configuration, that requires the less amount of the network source so it had better energy efficiency when compared with $3 \times 2$ and $4 \times 2$ antenna configuration. The cooperative MIMO scheme appears to be preferable than SISO schemes, but it is very much prone towards channel estimation errors and precise transmission synchronization remains highly essential for MIMO system.

\section{ACKNOWLEDGEMENTS}

The authors appreciated the support provided by school of Electronics Engineering, KIIT University, Bhubaneswar for the technical assistances.

\section{REFERENCES}

[1]. S. Mishra, H. Thakkar, " Features of WSN and Data Aggregation techniques in WSN : A Survey", International Journal of Engineering and Innovative Technology, vol. 1, April ,2011.

[2]. A. Paulraj, R. Nabar, and D. Gore, "Introduction to Space Time Wireless Communications", Cambridge, U.K Cambridge Univ. Press, 2003.

[3]. S. Cui, A. J. Goldsmith, and A. Bahai, "EnergyEfficiency of MIMO and Cooperative MIMO Techniques in sensor networks," IEEE journal on selected areas in communication, vol.22, August 2006.

[4]. S. Cui, A. J. Goldsmith, and A. Bahai "Energyconstrained modulation optimization," IEEE transactions on wireless communications, vol. 4, NO. 5, September 2005.

[5]. S. Cui, A. J. Goldsmith, and A. Bahai, "Modulation optimization under energy constraints," in Proc. ICC'03, AK, May 2003.

[6]. S. K. Jayaweera, "Energy analysis of mimo techniques in wireless sensor networks ," in 38th Annual Conference on Information Science sand System, Princeton University, USA, March2004.

[7]. Duc Nguyen, Olivier Berder ,Olivier Sentieys, "Cooperative MIMO schemes optimal selection for wireless sensor networks " IEEE, 2007.

[8]. Vibhav Kumar Sachan1,Syed A. Imam,M. T. Beg, " Design of Energy-Efficient Wireless Sensor Networks
Using Cooperative MIMO Techniques ", International Journal of Electronics Engineering, 3 (2), 2011.

[9]. Yongxian Song , Rongbiao Zhang , Zhuo Shen,"Analysis of Energy Consumption of Virtual MIMO Wireless Sensor Network", Journal of Networks, VOL. 7, December 2012 .

\section{BIOGRAPHIES}

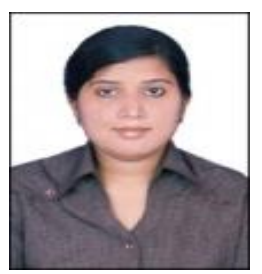

Meethu Abraham is pursing M.Tech in Communication System Engineering from KIIT University, Bhubaneswar, India. She had obtained B.Tech in Electronics and Communication Engineering from Maharshi Dayanand University, Rohtak, India in the year 2013. She has published paper in IJCA.

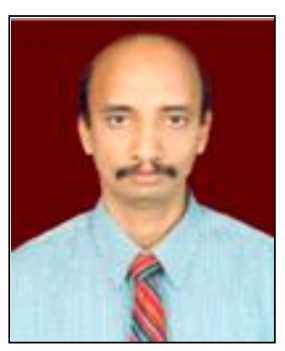

Dr. Sudhansu Sekhar Singh has received a $\mathrm{PhD}$ in Engineering (Mobile Communication) from Jadavpur University, Kolkata, India and M.E. in Electronic System and Communication Engineering from NIT Rourkela, India. He is working as a Professor in School of Electronics Engineering, KIIT University, Bhubaneswar, India. $\mathrm{He}$ is also the academic council member and board of studies member of different schools of the university. More than forty five publications in International journals and reputed international conference proceedings are to his credit. He has also guided more than twenty PG thesis and examined several of doctoral dissertations. His broad research area includes but not certainly limited to wireless and mobile communication, multicarrier CDMA, MIMO-OFDM, Wireless Sensor Networks. 